\title{
Hydraulic Ripper Computer Modeling and Simulation
}

\author{
Yanping Huang, Guoping Yang \\ Shanghai University of Engineering Science, Shanghai, China \\ Email: $1040077082 @ q q . c o m$
}

How to cite this paper: Huang, Y.P. and Yang, G.P. (2018) Hydraulic Ripper Computer Modeling and Simulation. Open Access Library Journal, 5: e4495.

https://doi.org/10.4236/oalib.1104495

Received: March 11, 2018

Accepted: March 27, 2018

Published: March 30, 2018

Copyright () 2018 by authors and Open Access Library Inc.

This work is licensed under the Creative Commons Attribution International License (CC BY 4.0).

http://creativecommons.org/licenses/by/4.0/

\begin{abstract}
It's very useful to put the virtual prototype technology into hydraulic ripper, just for improving the product research and development efforts. A certain type of hydraulic ripper was simulated and calculated using CATIA and ADAMS software, the simulation system is set up, and the relevant parameters of hydraulic ripper are gotten; on this basis, a prototype model is compared with the reality. At the same time, a comparative analysis of simulation results and real results under certain conditions is used to verify the rationality of the prototype model, and the reference value of its products must build a good development platform for hydraulic ripper.
\end{abstract}

\section{Subject Areas}

Mechanical Engineering

\section{Keywords}

Hydraulic Ripper, ADAMS, CATIA, Modeling Simulation

\section{Introduction}

Hydraulic ripper is a kind of broken hammer in recent years, domestic research in this area less, but some domestic enterprises and universities in this area carried out a systematic study. However, with the research of virtual prototyping, it is more and more important to analyze the structure and system of Hydraulic ripper. In this paper, the research of Hydraulic ripper is carried out by using CATIA for 3D modeling, and then introduced into the simulation software ADAMS. Then, the parameters are added and simulated by using the View module in ADAMS of multi-body system dynamics software. Through this series, the simulation results are compared with the real results to verify the rationality and validity of the data and simulation, and the feasibility of applying the 
virtual prototyping technology to the research and development of Hydraulic ripper, which provides a reference for the research of virtual prototyping technology of Hydraulic ripper.

\section{Basic Structure and Working Principle of Hydraulic Ripper}

The hydraulic ripper is composed of an exciter, a rod mechanism, a shock-absorbing mechanism and a crushing mechanism composed of a knife row and a bucket, wherein the exciter is arranged on the crushing mechanism and is connected with it, and the crushing mechanism is connected with the rod mechanism The cushioning mechanism is mounted on the upper surface of the crushing mechanism mounting table [1]. Its working principle is through the excavator to provide high-speed hydraulic oil, pipeline from the Hydraulic ripper connected to the hydraulic motor, hydraulic motor through the spline and the shaft connected with the eccentric block gear through the key and the shaft, hydraulic The motor rotates the drive shaft and the shaft drives the drive wheel of the eccentric block gear. The active eccentric wheel drives the driven eccentric wheel to rotate, and the pair of meshing with the eccentric gear rotates, resulting in eccentric forces superimposed in the vertical direction, The direction of each other to offset each other, through the upper and lower vibration gear and knife row connected to the broken force to the bucket teeth, in this role under the cracked stones to achieve the crushing effect [2].

\section{Hydraulic Ripper Model Established}

\subsection{Establishment of 3D Model in CATIA}

According to a Hydraulic ripper, in the CATIA environment to establish three parts of the three parts of the model and assembly, as shown in Figure 1 is a Hydraulic ripper explosion map, through the explosion map can be a good show about the relationship between the various parts of Hydraulic ripper, this simple design language to a large extent facilitate the exchange of technical personnel, reducing the various aspects of the error [3] [4].

\subsection{Establishment of Virtual Prototype Model in ADAMS}

Before modeling with the software ADAMS software module, the construction of the mechanical system model must be carried out. Some of the common constraints and forces, geometric models and a series of settings, and then parametric analysis.

1) Hydraulic ripper into ADAMS software

According to CATIA software in a burst map, the vibration box and the case, the air spring to. Part of the form into the ADAMS/View, first determine the location of the shock box, and then in accordance with the assembly to determine the location of other parts, the final assembly A Virtual Prototyping Model in ADAMS Software. At the same time will be assembled into a number of 


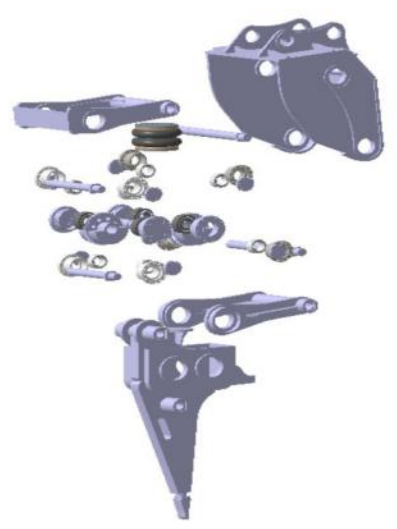

Figure 1. Hydraulic ripper explosion map.

modules into the Adams, respectively, for example, into the ADAMS shell, respectively, attached to the quality, moment of inertia, moment of inertia, centroid coordinates (Figure 2).

2) Motion constraint add

After importing into ADAMS through CATIA software, in order to ensure that the simulation is performed correctly, constraints must be added to constrain the motion direction and motion of the moving parts.

1) Contact constraints

There are two constraints in this model: two gears with eccentric blocks, bucket teeth and broken objects. The impact function model is expressed by displacement, velocity, and contact force. The characteristics of this method are applicable to all cases of continuous and discontinuous type of contact force [5]. According to the characteristics of Hydraulic ripper, the impact function model is selected to define the contact constraint of this model.

2) Rotate the sub-constraint

The rotary pair is often used for two objects so that the member 1 rotates relative to the member 2 for rotation. The simplified Hydraulic ripper has six rotary pairs, in which the housing is connected with the pull-up bracket, the housing and the pull-up the bracket and the shock box, the pull-down bracket and the shock box, the vibration box and the active component, the vibration box and the driven component, all six are the rotation vice.

3) Other constraints

The model of the Hydraulic ripper is simulated in the course of the stone fixed on the earth, the chassis selection fixed pair of fixed and the earth fixed, shock box, knife row, bucket teeth selection associate association, in this process for the air spring The treatment is handled according to a contact spring.

3) Drive the addition

The actual working chamber of the Hydraulic ripper is realized by the input torque of the hydraulic motor. In the simulation, we use the addition speed of the direct shaft instead of the hydraulic motor speed. The speed we use the step function STEP () to express, in the actual conditions, the high-frequency 


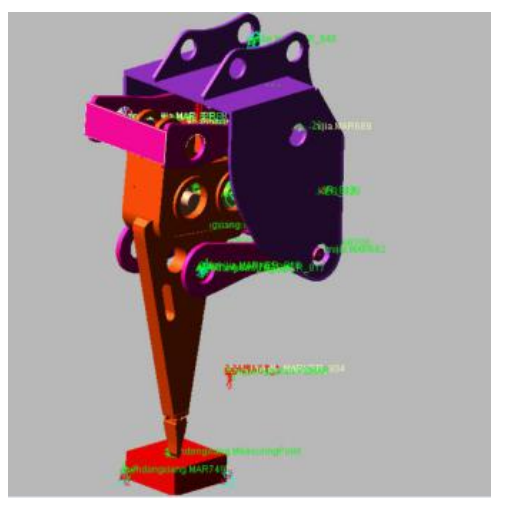

Figure 2. The virtual prototype model of Hydraulic ripper in ADAMS.

hydraulic breaker hammer speed of $1500 \mathrm{r} / \mathrm{min}$ to $1800 \mathrm{r} / \mathrm{min}$ when the best blow to break the effect, we choose this Range of the study, $1800 \mathrm{r} / \mathrm{min}$ step function for the STEP (time, 0, 0, 0.05, $10800 \mathrm{~d}$ ).

4) Establish measurement and simulation control settings

The purpose of the virtual prototype model is to obtain a series of data on the acceleration and velocity, displacement and energy of the bucket teeth of the Hydraulic ripper. We set the simulation time $2 \mathrm{~s}$, simulation steps 2000 , set the Figure 3.

\section{Simulation Results Analysis}

After the simulation is completed, we can call ADAMS/Post Processor post-processing module to view and analyze the simulation data obtained in this simulation, through simulation, we got the hydraulic ripper bucket teeth speed, acceleration, displacement and energy of the dynamic curve, the detailed results are shown in Figures 4-8.

As can be seen from Figure 4, the stroke in the direction of gravity is 35.1 $\mathrm{mm}$, the process of running is stable, has been stable within a certain range, the displacement of the extreme position of the slight fluctuations, this is because the high frequency Hydraulic crusher in the process of breaking the stones, the stones will be broken, so there will be displacement deviation.

As can be seen from Figure 5, the displacement stroke in the direction of the tool nose is $0.498 \mathrm{~mm}$, less than $1 \mathrm{~mm}$, and it is in a steady state at the limit position. There is no fluctuation in the displacement.

As can be seen from Figure 6, the period of motion is $\mathrm{T}=0.0375 \mathrm{~s}$, and the number of blows per minute is 1600 times, which is in line with the number of strikes per minute $1500-1740$ provided by the manufacturer. In the course of the movement, firstly the bucket accelerates at an acceleration of $69.57 \mathrm{~m} / \mathrm{s}^{2}$. After the collision, the maximum acceleration reaches $394.05 \mathrm{~m} / \mathrm{s}^{2}$. It can be seen that when the acceleration is greater than After $247.56 \mathrm{~m} / \mathrm{s}^{2}$, the combination of the knife row and the vibration box will be deformed, so we should avoid excessive acceleration. 


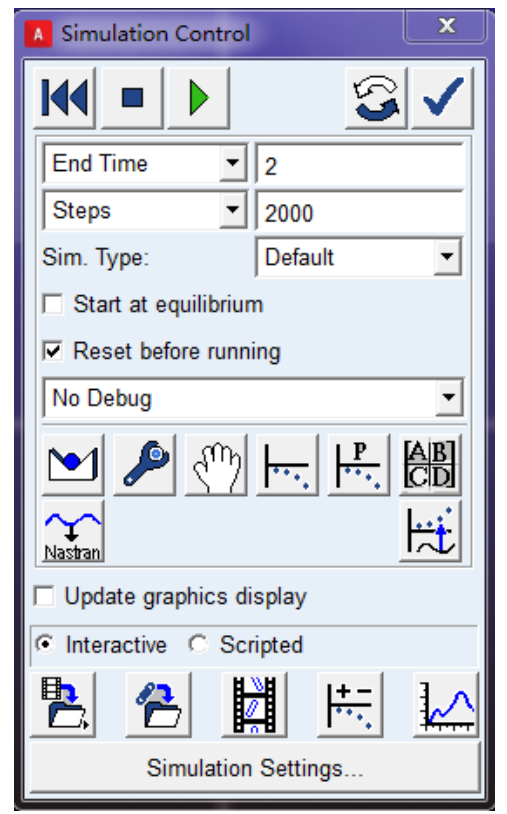

Figure 3. Simulation control settings.

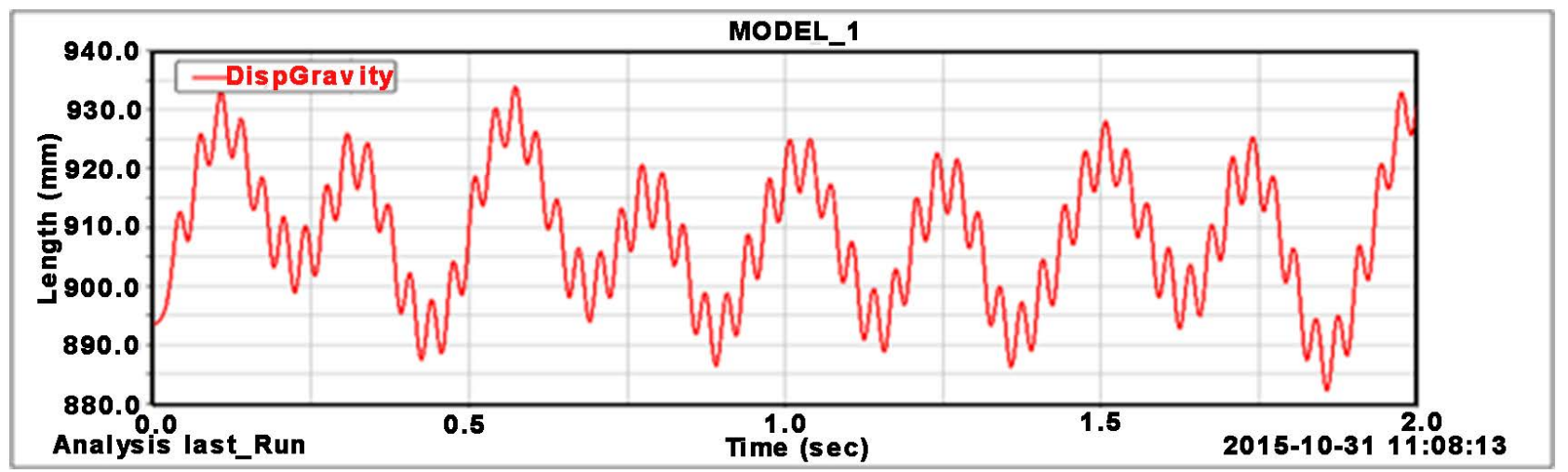

Figure 4. Bucket teeth displacement curve in the direction of gravity.

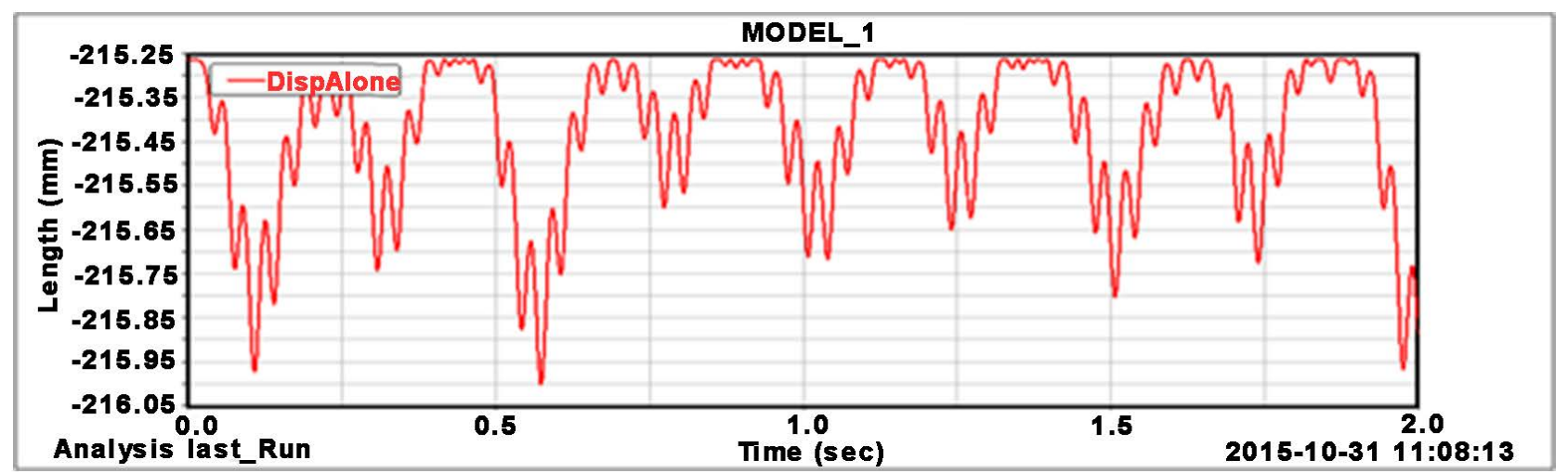

Figure 5. Bucket teeth displacement curve in the direction of knife board.

It can be seen from Figure 7 that when the operation is started, the acceleration in the direction of the knife is relatively small, the acceleration is $1.285 \mathrm{~m} / \mathrm{s}^{2}$, the maximum acceleration is $8.642 \mathrm{~m} / \mathrm{s}^{2}$, the acceleration is for the rock 


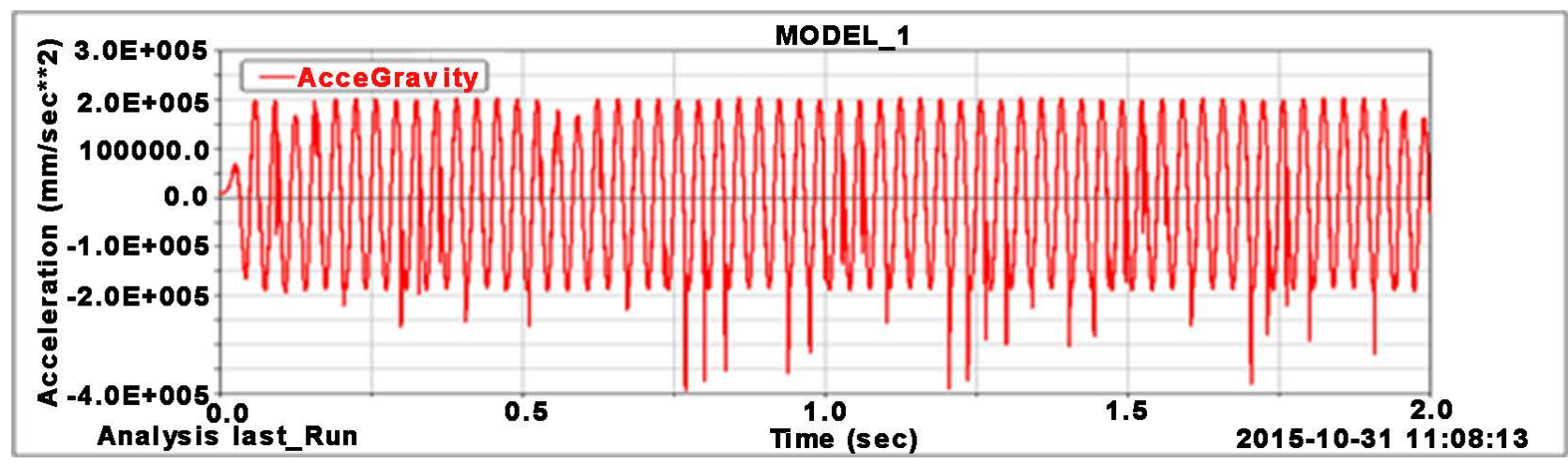

Figure 6. Bucket teeth acceleration curve in the direction of gravity.

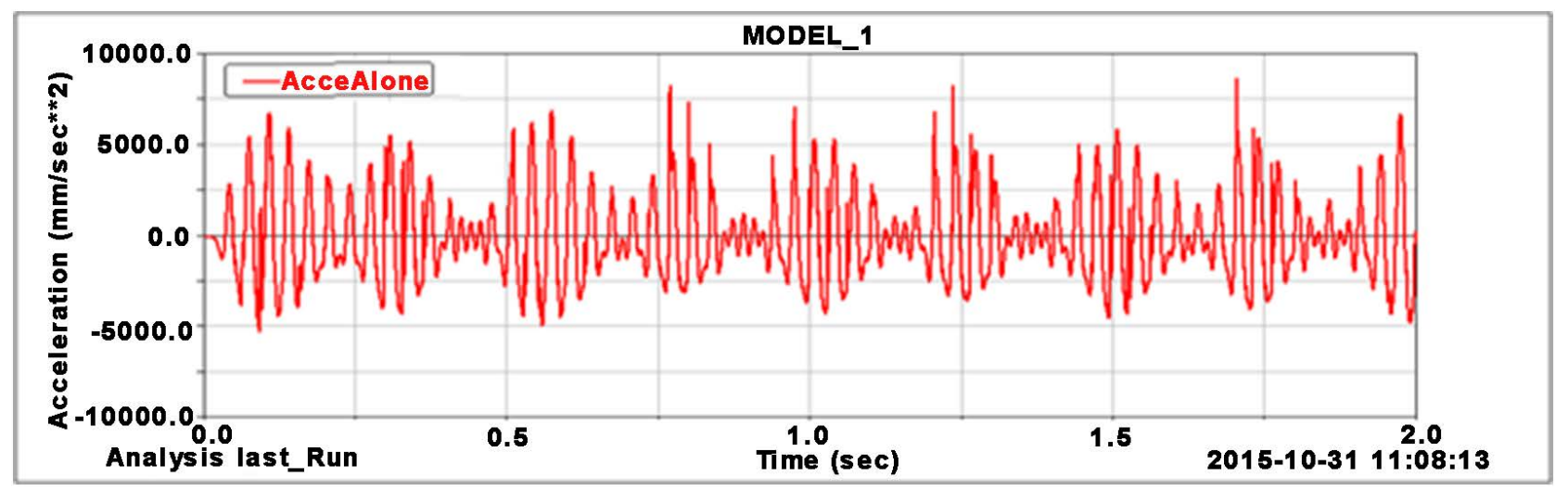

Figure 7. Bucket teeth acceleration curve in the direction of knife board.

Crushing little effect, so we can ignore the impact of its acceleration on the performance of Hydraulic ripper.

It can be seen from Figure 8, the maximum impact energy of Hydraulic ripper can reach $1817.7 \mathrm{~J}$, at the moment of start, Hydraulic ripper energy reached $843.6 \mathrm{~J}$, in the subsequent work process, the basic distribution of energy, To a certain extent, a true reflection of the Hydraulic ripper the real working state, the parameters have a certain reference value.

As can be seen from Table 1, the virtual prototype model of the simulation results and the real results there are still many gaps, these may be the virtual prototype in the simulation process abandoned part of the useless parts and simplified, and has not yet taken into account The impact of lubricants on the organization, if these factors can be taken into account, the simulation results and the difference between the official data should not be now big.

\section{Conclusion}

Virtual prototyping technology uses the computer's powerful computing power, through the creation of three-dimensional model, at the same time for the product after the work conditions for the kinematics and dynamics in the simulation, analysis of mechanical movement and system simulation parameters, as the basis for the application to the product improvement and development 


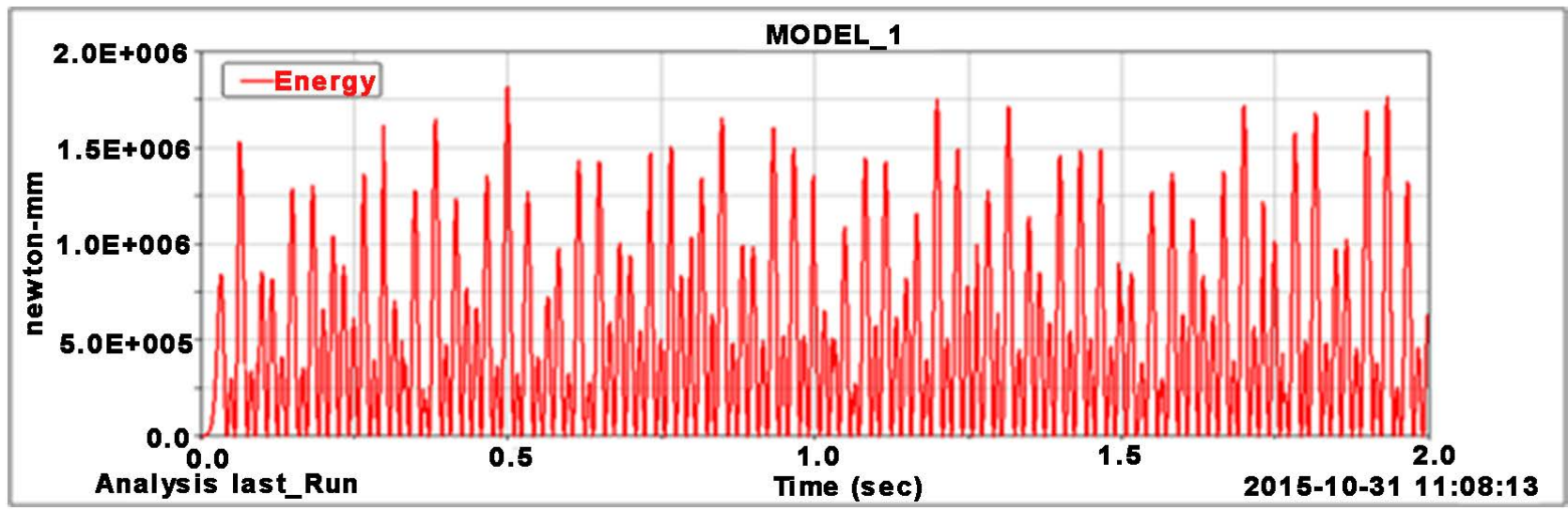

Figure 8. Hydraulic ripper impact energy curve.

Table 1. Data comparison table.

\begin{tabular}{ccccc}
\hline Content & Bucket teeth trip $(\mathrm{mm})$ & Impact speed $(\mathrm{m} / \mathrm{s})$ & Hit frequency $(\mathrm{Hz})$ & Maximum impact energy $(\mathrm{J})$ \\
\hline Official data & $\leq 50$ & $\leq 2.3$ & $25-29$ & 2500 \\
Simulation data & 35.1 & 1.1 & 26.6 & 1817.7 \\
\hline
\end{tabular}

process, and to provide the basis for this basis [6]. The application of virtual prototyping technology in hydraulic ripper is not only theoretical, but also has great practical value. Hydraulic ripper was simulated and analyzed in ADAMS software to obtain a series of parameters and the movement characteristics curve of the bucket tooth. Based on the reference, the displacement, acceleration, and frequency movement rules of the bucket teeth were analyzed. Based on the parameters curve of the production punching skill, the simulation results obtained are close to the parameters provided by the manufacturer, which verifies the correctness of hydraulic ripper model under certain assumptions.

\section{References}

[1] Cao, Y. and Fan, Y.X. (2012) A High-Frequency Hammer: China, 202590877U. 2012-12-12.

[2] Wang, K.L., Yang, G.P., Hu, K.J., et al. (2015) High Frequency Hammer Development Status and Research. Mining Machinery, No. 4, 1-4.

[3] Wang, L. and Yang, G.P. (2011) Computer Modeling and Simulation of Hydraulic Breaking Hammer. Machine Tool \& Hydraulics, 39, 88-92.

[4] Yang, G.P., Liu, X.J., Gao, J.H., et al. (2011) Improved Design of Hydraulic Impactor Based on Virtual Prototyping Technology. Mechanical Design and Manufacture, No. 8, 22-24.

[5] Pei, W.C., Li, Y.G., Li, Y.H., et al. (2008) Impact Model Based on Virtual Prototyping Technology-The Impact Model of ADAMS. Journal of HeBei Polytechnic University (Natural Science Edition), 30, 59-63.

[6] Wang, L. (2010) Hydraulic Crusher Virtual Prototype Technology Research. Master Thesis, Shanghai Engineering University, Shanghai. 